\title{
Analysis of Surgical Pathology Data in the HIRA Database: Emphasis on Current Status and Endoscopic Submucosal Dissection Specimens
}

\author{
Sun-ju Byeon · Woo Ho Kim \\ Department of Pathology, Seoul National \\ University Hospital, Seoul, Korea
}

\begin{abstract}
Background: In Korea, medical institutions make claims for insurance reimbursement to the Health Insurance Review and Assessment Service (HIRA). Thus, HIRA databases reflect the general medical services that are provided in Korea. We conducted two pathology-related studies using a HIRA national patient sample (NPS) data (selection probability, 0.03). First, we evaluated the current status of general pathologic examination in Korea. Second, we evaluated pathologic issues associated with endoscopic submucosal dissection (ESD). Methods: The sample data used in this study was HIRA-NPS-2013-0094. Results: In the NPS dataset, 163,372 pathologic examinations were performed in 103,528 patients during the year 2013. Considering sampling weight (33.3), it is estimated that 5,440,288 $(163,372 \times 33.3)$ pathologic examinations were performed. Internal medicine and general surgery were the most common departments requesting pathologic examinations. The region performing pathologic examinations were different according to type of medical institution. In total, 490 patients underwent ESD, and 43.4\% (213/490) underwent ESD due to gastric carcinoma. The results of the ESD led to a change in disease code for $10.5 \%$ (29/277) of non-gastric carcinoma patients. In addition, 21 patients (4.3\%) underwent surgery following the ESD. The average period between ESD and surgery was 44 days. Conclusions: HIRA sample data provide the nation-wide landscape of specific procedure. However, in order to reduce the statistical error, further studies using entire HIRA data are needed.
\end{abstract}

Key Words: Statistics; Sample size; Pathology; Surgery
In Korea, all medical institutions claim insurance reimbursements for services to the Health Insurance Review and Assessment Service (HIRA). Thus, HIRA databases reflect the general medical services provided in Korea. Since the entire HIRA database is too big to analyze, HIRA provides a specific set of data according to researcher's requests. HIRA also provides some relatively small sized data sets (statistically extracted, anonymized, and annualized) for research and public purposes. ${ }^{1}$ These sample data included national patient sample (NPS), a national inpatient sample, an adult patient sample (65 years or older), and a pediatric patient sample. To the best of our knowledge, this is the first pathology-related analysis using HIRA data.

We conducted two pathology-related studies using HIRANPS data. First, we analyzed the rate of surgical pathologic examinations in Korea. After Kamegoro Inamoto introduced pathology to Korea in 1914, pathologic examinations have played a major role in medical services for the improvement of overall care. ${ }^{2}$ However, research on the frequency of pathologic examinations in this nation as a whole have not been performed due to a restriction on the sharing of personal medical records.
Next, we analyzed the pathologic results of endoscopic submucosal dissection (ESD) specimens. ESD is considered as an initial treatment modality for early and localized gastric carcinoma or benign epithelial neoplasm. ${ }^{3}$ We evaluated several ESD-related parameters. After ESD and pathologic examination, surgical treatment is called for in those cases that show tumor cells in the resection margins, endolymphatic tumor emboli, or submucosal invasion. ${ }^{4}$ Several papers have been published on this subject, but most of them are from a single institute and so do not incorporate the data of those patients who later went on to undergo additional surgery in different hospitals. ${ }^{5,6}$ One of the advantageous features of the HIRA database is that patients can be tracked through several medical institutions, thereby enabling the study of a more complete data set including those that received secondary treatment in one hospital after ESD in another hospital.

\section{MATERIALS AND METHODS}

The sample data used in this study was HIRA-NPS-2013-0094. 
This sample was composed of 26 text files, and the total file size was 16.6 gigabytes (GB). The HIRA data tables were composed of five main tables (general specification, healthcare services, diagnosis information, prescriptions, and general information about the medical institutions). Each table was conjoined with proper claim numbers or medical institution numbers. The disease codes were based on the Korean Standard Classification of Disease (KCD). Specifications of the computer that was used to analyze the data were as follows: central processing unit (CPU), i3-2120 $3.30 \mathrm{GHz}$ (Intel, Santa Clara, CA, USA); 32 GB main memory; operating system (OS), Ubuntu 14.04.3 long-term support (LTS); and R 3.2.2 analysis software. The pathologic examination codes used in this study are summarized in Table 1 . The sample data contained 22,344,536 claims in 1,361,717 patients (selection probability, 0.03 ; sampling weight, 33.3).

Table 1. Pathologic examination claim codes using in this study

\begin{tabular}{|c|c|}
\hline Histologic examination & Code \\
\hline Biopsy: 1-3 pieces/4-6 pieces/7-9 pieces/10-12 pieces/more than 13 pieces & C5911/C5912/C5913/C5914/C5915 \\
\hline Resected specimen requiring gross sectioning (non-malignant): paraffin blocks $\leq 6 /$ paraffin blocks $\geq 7$ & C5916/C5917 \\
\hline \multicolumn{2}{|l|}{ Resected specimen for malignant tumor requiring gross sectioning } \\
\hline With lymph node dissection: paraffin blocks $\leq 20 /$ paraffin blocks $\geq 21$ & C5918/C5919 \\
\hline Without lymph node dissection: paraffin blocks $\leq 15 /$ paraffin blocks $\geq 16$ & C5500/C5504 \\
\hline Histologic mapping of tumor: with lymph node dissection/without lymph node dissection & C5505/C5508 \\
\hline $\begin{array}{l}\text { Emergency histopathologic examination during surgery (frozen section): 1-2 specimens/3-6 specimens/ } \\
\text { more than } 7 \text { specimens }\end{array}$ & C5511/C5512/C5513 \\
\hline Tissue immunofluorescent microscopic examination: lgG/lgA/lgM/lgE/C3/C4/HbsAg/fibrinogen/others & $\begin{array}{l}\text { C5541/C5542/C5543/C5544/C5545/C5549/ } \\
\text { C5546/C5547/C5548 }\end{array}$ \\
\hline Tissue electron microscopy & C5550 \\
\hline $\begin{array}{l}\text { Enzyme histochemistry: ATPase-pH 9.4/ATPase-pH 4.9/NADH/acetylcholinesterase/ } \\
\text { chloroacetate esterase/others }\end{array}$ & C5561/C5562/C5563/C5564/C5565/C5566 \\
\hline Immunohisto(cyto)chemistry & C5575/C5575006 \\
\hline Cervicovaginal smear/Liquid-based cervicovaginal cytology & C5920/CX541 \\
\hline $\begin{array}{l}\text { Body fluid: general/cytospin/cell block after cytopathologic examination/liquid-based body fluid } \\
\text { cytopathology }\end{array}$ & C5930/C5931/C5940/CZ521 \\
\hline Aspiration/Aspiration and cell block & C5941/C5942 \\
\hline Liquid-based aspiration cytopathology/Liquid-based aspiration cytopathology and Cell block & C5943/C5944 \\
\hline Flurescence in situ hybridization: HER2 gene & C5967/C5967006 \\
\hline Silver in situ hybridization: HER2 gene & CZ968/CZ968006 \\
\hline
\end{tabular}

HBsAg, hepatitis B surface antigen.

Table 2. Pathologic examinations claims and requesting medical institutions according to administrative districts (sort based on the total number of claims)

\begin{tabular}{lcccrr}
\hline Administrative district & Tertiary hospital & General hospital & Other institutions & Total & Total estimates \\
\hline Seoul & 25,547 & 16,164 & 12,000 & 53,711 & $1,788,576$ \\
Gyeonggi-do & 1,632 & 16,203 & 10,905 & 28,740 & 957,042 \\
Busan & 3,276 & 4,877 & 5,135 & 13,288 & 442,490 \\
Daegu & 1,164 & 5,933 & 5,185 & 12,282 & 408,991 \\
Incheon & 2,545 & 2,377 & 2,465 & 7,387 & 245,987 \\
Gyeongsangnam-do & 760 & 3,561 & 3,053 & 7,374 & 245,554 \\
Gwangju & 1,211 & 2,438 & 1,910 & 5,559 & 185,115 \\
Daejeon & 970 & 2,300 & 1,781 & 5,051 & 168,198 \\
Jeollabuk-do & 857 & 1,935 & 2,162 & 4,954 & 164,968 \\
Gyeongsangbuk-do & - & 2,252 & 2,638 & 4,890 & 162,837 \\
Jeollanam-do & - & 2,655 & 1,621 & 4,276 & 142,391 \\
Chungcheongnam-do & - & 2,024 & 1,861 & 3,885 & 129,371 \\
Gangwon-do & 850 & 1,791 & 1,046 & 3,687 & 122,777 \\
Chungcheongbuk-do & 573 & 1,178 & 1,611 & 3,362 & 111,955 \\
Ulsan & - & 1,561 & 1,799 & 3,360 & 111,888 \\
Jeju & - & 1,135 & 380 & 1,515 & 50,450 \\
Sejong-si & - & - & 58 & 1,698 \\
Total & 39,385 & 68,384 & 55,603 & 163,372 & $5,440,288$ \\
\hline
\end{tabular}




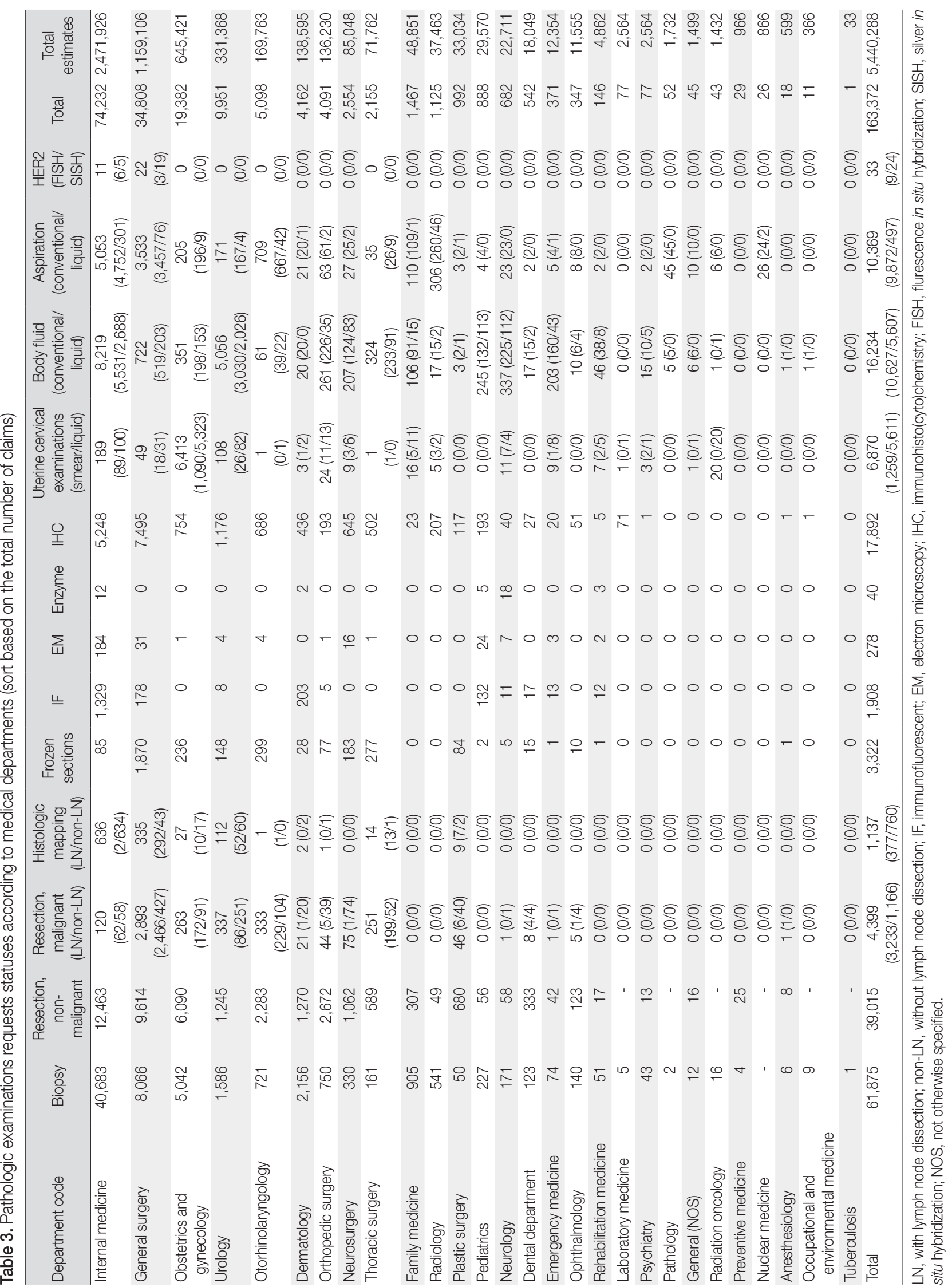




\section{RESULTS}

\section{Pathologic examination statistics in Korea}

In the year 2013, 163,372 pathologic examinations were performed in 103,528 patients (45,897 men and 57,631 women). The mean and median ages of the patients were 51.8 and 53 years, respectively. The skewness and kurtosis of patient age were -0.251 and 2.775 , respectively. The pathologic examination data and medical institutions according to administrative district are summarized in Table 2. The total numbers of medical institutions according to administrative district are summarized in Appendix 1. In total, $43.5 \%$ of the tertiary hospitals (10/23), $15.6 \%$ of the general hospitals (48/307), and $23.8 \%$ of the other institutions $(12,090 / 50,868)$ were located in Seoul. In addition, $32.9 \%$ of the surgical pathologic examinations $(53,711 / 163,372)$ were requested in medical institutions located in Seoul.

Almost all medical and dental departments requested pathologic examinations (Table 3). Internal medicine (74,232, 45.4\%), general surgery $(34,808,21.3 \%)$, obstetrics and gynecology $(19,382,11.9 \%)$, and urology $(9,951,6.1 \%)$ were the most common medical departments $(84.7 \%)$ requesting pathology examinations. A small subset $(38 / 17,892,0.2 \%)$ of immunohistochemical (IHC) stains were not interpreted by qualified doctors (not claimed as "C5575006") (data not shown). Claims for ace- tylcholinesterase (C5564) and chloroacetate esterase (C5565) examinations were not found in our data.

Among pathologic examination-associated claims, 162,002 examinations $(99.2 \%$ ) had proper claim codes (examination codes 09 and sub-code 01 (performed in their own institutions) or 02 (performed in outside institutions). Among pathologic examinations, $65.8 \%$ were performed in tertiary or general hospitals (39,385 and 67,225 cases, respectively) (Table 4). Almost all examinations claimed by tertiary hospitals were performed in their institute $(39,349 / 39,385,99.9 \%)$, and $85 \%$ of examinations claimed by general hospitals were performed $(57,163 / 67,225)$ in their institute. The other medical institutions claimed considerable pathologic examinations performed by outside services $(52,214 / 55,392,94.3 \%)$.

According to the claims, 76,016 pathologic examinations $(44.7 \%)$ were performed due to malignancy (disease code "C") or non-malignant tumorous conditions (disease code "D00-D48”) (Table 5). IHC stains were more frequently performed in tumorous conditions $(15,336 / 17,892,85.7 \%)$. Pathologic examination of biopsy specimens was performed more frequently in non-tumorous conditions $(42,661 / 61,875,68.9 \%)$.

\section{ESD-related statistics}

In total, 509 ESDs were performed in 490 patients (341 males

Table 4. Pathologic examination performing places according to medical institutions

\begin{tabular}{lcccr}
\hline Institution & Tertiary hospital & General hospital & Other medical institutions & Total \\
\hline In own hospital & $39,349(99.9)$ & $57,163(85.0)$ & $3,178(5.7)$ & $99,690(61.5)$ \\
Outside services & $36(0.1)$ & $10,062(15.0)$ & $52,214(94.3)$ & $62,312(38.5)$ \\
Total & $39,385(100)$ & $67,225(100)$ & $55,392(100)$ & $162,002(100)$ \\
\hline
\end{tabular}

Values are presented as number (\%).

Table 5. Disease codes of patients at time of pathologic examinations requests

\begin{tabular}{lccc}
\hline & Tumorous condition & Non-tumorous condition & Total \\
\hline Biopsy & 19,214 & 42,661 & 61,875 \\
Resection, non-malignant & 14,900 & 24,115 & 39,015 \\
Resection, malignant (LN/non-LN) & $4,333(3,203 / 1,130)$ & $66(30 / 36)$ & $4,399(3,233 / 1,166)$ \\
Histologic mapping (LN/non-LN) & $1,045(377 / 668)$ & $92(0 / 92)$ & $1,137(377 / 760)$ \\
Frozen sections & 3,041 & 281 & 3,322 \\
IF & 213 & 1,695 & 1,908 \\
EM & 51 & 227 & 278 \\
Enzyme & 0 & 40 & 40 \\
IHC & 15,336 & 2,556 & 17,892 \\
Uterine cervical examinations (smear/liquid) & $3,199(554 / 2,645)$ & $3,671(705 / 2,966)$ & $6,870(1,259 / 5,611)$ \\
Body fluid (conventional/liquid) & $7,137(4,118 / 3,019)$ & $9,097(6,509 / 2,588)$ & $16,234(10,627 / 5,607)$ \\
Aspiration (conventional/liquid) & $4,514(4,234 / 280)$ & $5,855(5,638 / 217)$ & $10,369(9,872 / 497)$ \\
HER2 (FISH/SISH) & $33(9 / 24)$ & $0(0 / 0)$ & $33(9 / 24)$ \\
Total & 73,016 & 90,356 & 163,372 \\
\hline
\end{tabular}

LN, with lymph node dissection; non-LN, without lymph node dissection; IF, immunofluorescent; EM, electron microscopy; IHC, immunohisto(cyto)chemistry; FISH, flurescence in situ hybridization; SISH, silver in situ hybridization. 
and 149 females) in 109 medical institutions. The median age of the patients was 66 years (age, 29 to 89 years; 1st quantile, 58 years; 3 rd quantile, 71.75 years). The majority of patients ( $\mathrm{n}=$ 472) underwent one ESD; 17 patients underwent two ESDs (10 patients underwent simultaneous ESD, while seven patients underwent ESDs at different times), and one patient underwent three ESDs (two ESDs at the same time).

The disease codes noted when patients underwent ESD were as follows: $\mathrm{C} 16$ (malignant neoplasm of stomach), 213 patients (43.5\%); non-C16, 277 patients (56.5\%); D00.2 (carcinoma in situ of stomach), 17 patients; D13.1 (benign neoplasm of stomach), 226 patients; D13.9 (benign neoplasm of ill-defined site within the digestive system), one patient; and other, 33 patients. Disease codes changed after the ESD in 10.5\% of the non-C16 patients (29/277): D00.2 to C16, seven patients; D13.1 to D00.2, three patients; D13.1 to C16, 18 patients; and D13.9 to C16, one patient (Table 6).

Twenty-one patients $(21 / 490,4.3 \%)$ underwent gastrectomy following ESD, and all of these patients underwent ESD for only once. Fifteen patients received surgery at the same medical insti-

Table 6. Changes of disease codes after endoscopic submucosal dissections

\begin{tabular}{lccc}
\hline \multirow{2}{*}{$\begin{array}{c}\text { Disease code } \\
\text { at ESD }\end{array}$} & \multicolumn{3}{c}{ Disease code after ESD } \\
\cline { 2 - 4 } & Unchanged & Changed to C16 & Changed to D00.2 \\
\hline D00.2 & 10 & 7 & - \\
D13.1 & 205 & 18 & 3 \\
D13.9 & 0 & 1 & 0 \\
Others & 33 & 0 & 0 \\
Total & 248 & 26 & 3 \\
\hline
\end{tabular}

ESD, endoscopic submucosal dissections. tution where ESD was performed, and six patients received surgery at different medical institutions. The mean time between ESD and surgery was 44 days. Two patients changed diagnosis (benign to malignant) after ESD. One patient underwent ESD and surgery during the same hospitalization period.

For further analysis, 472 patients who underwent ESD for only once were selected (Table 7) (C16, 202 patients; D00.2, 17 patients; D13.1, 220 patients; D13.9, one patient; other, 32 patients). In total, $70.0 \%$ of these pathologic examinations (329/ 472) were requested for histologic mapping (C5508). IHC studies were performed in 22.5\% of ESDs (106/472). Approximately one-third of the gastric cancer specimens $(66 / 202,32.7 \%)$ and $15.0 \%$ of the gastric benign neoplasm specimens (33/220) were subject to IHC studies.

\section{DISCUSSION}

In this study, we examined nation-wide statistics regarding surgical pathologic examination. Considering sampling weight, we estimate that 5,440,288 $(163,372 \times 33.3)$ pathologic examinations were performed in Korea in 2013. We also surveyed pathologic examinations according to administrative district, requesting department, type of medical institutions, and patient conditions. These data will be useful for future planning and allocation of resources in the field of pathology and for the Korean Society of Pathologists.

There have been several reports regarding diagnostic discrepancies between endoscopic forceps biopsy and ESD, as well as between ESD and surgery. Recently, two large, single-center, retrospective studies revealed that 22.8\% (465/2,041) and 31.7\%

Table 7. Pathologic examination codes and number of immunohistochemical stains according to diseases codes at endoscopic submucosal dissections

\begin{tabular}{|c|c|c|c|c|c|c|}
\hline & C16 & D00.2 & D13.1 & D13.9 & Others & Total \\
\hline \multicolumn{7}{|c|}{ Pathologic examination codes } \\
\hline C5500 & 6 & 0 & 5 & 0 & 0 & 11 \\
\hline C5916 & 16 & 3 & 51 & 0 & 2 & 72 \\
\hline C5917 & 22 & 11 & 25 & 0 & 2 & 60 \\
\hline Total & 202 & 17 & 220 & 1 & 32 & 472 \\
\hline \multicolumn{7}{|c|}{ No. of immunohistochemical stains } \\
\hline 0 & 136 & 14 & 187 & 1 & 28 & 366 \\
\hline 1 & 42 & 1 & 25 & 0 & 1 & 69 \\
\hline 2 & 12 & 0 & 5 & 0 & 2 & 19 \\
\hline 3 & 2 & 1 & 3 & 0 & 1 & 7 \\
\hline 4 & 2 & 1 & 0 & 0 & 0 & 3 \\
\hline 5 & 3 & 0 & 0 & 0 & 0 & 3 \\
\hline Total & 202 & 17 & 220 & 1 & 32 & 472 \\
\hline
\end{tabular}


$(587 / 1,850)$ of cases changed diagnosis after endoscopic resection in Asan Medical Center (Seoul, Korea) and Samsung Medical Center (Seoul, Korea), respectively. ${ }^{5,6}$ Our HIRA-NPS data revealed that only $10.9 \%$ of nation-wide cases experienced a change of diagnosis after ESD. HIRA-NPS data does not include detailed pathologic diagnosis information such as tumor size, tumor differentiations, dysplastic degrees, etc., so further analysis for clarifying such differences was limited. Shin et al ${ }^{7}$ reported that complete resection rates were not different according to absolute or expanded ESD indications, though their data were not HIRA data. Although HIRA-NPS data was not available for a sufficient number of ESD patients (509 ESDs in 490 patients) to allow for an accurate assessment, and only limited clinicopathological information was available, the problems associated with the relatively few number of patients can be overcome through further research using the raw HIRA data.

Histologic mapping of ESD specimens is recommended by The Gastrointestinal Pathology Study Group of the Korean Society of Pathologists. ${ }^{8}$ However, 30.2\% (143/472) of ESD specimens were not claimed as "C5508" (histologic mapping without lymph node dissection). Because precise histologic diagnosis of the ESD specimen is essential to treat patients, we suggest that nationwide surveys be conducted in order to assure quality of pathologic examination of ESD specimens. IHC staining was performed in 22.5\% (106/472) of ESD cases. Unlike tissue immunofluorescent microscopic examinations or enzyme histochemistries, HIRA data does not list the specific antibodies used for IHC stains. Thus, further analysis of IHC studies using HIRA data was limited.

The main limitations of our analysis are statistical issues based on probability sampling. Our estimates were calculated from statistically extracted data from one year. Further analysis using HIRA raw data will be needed to decrease the statistical errors and bias and to evaluate changes over time. During our analysis, we experienced many challenges; therefore, we provide advice and guidance for other researchers who would like to analyze the HIRA database.

(1) It is essential to understand the framework of the HIRA database. ${ }^{9}$ The HIRA data is intended for insurance claims and not for research. Detailed clinicopathological data are not provided. (2) Sample data are not appropriate for analyses requiring long-term follow-up. (3) Some claim codes have sub-codes (in most cases, additional charges by experts). (4) Disease codes and claims are not always accurate. (5) The analysis system should have at least $32 \mathrm{~GB}$ of main memory. (6) It is helpful to create relatively small data tables to decrease operation time. In the usual setting, R should use only one CPU core during calculation.

\section{Conflicts of Interest}

No potential conflict of interest relevant to this article was reported.

\section{REFERENCES}

1. Kim L, Kim JA, Kim S. A guide for the utilization of Health Insurance Review and Assessment Service National Patient Samples. Epidemiol Health 2014; 36: e2014008.

2. Chi JG. The establishment of hospital pathology in Korea. Korean J Pathol 1994; 28: 109-17.

3. Lee CK, Chung IK, Cho JY, et al. A survey on the indication for endoscopic submucosal dissection in early gastric cancer. Korean J Gastrointest Endosc 2009; 39: 78-84.

4. Song KY, Hyung WJ, Kim HH, et al. Is gastrectomy mandatory for all residual or recurrent gastric cancer following endoscopic resection? A large-scale Korean multi-center study. J Surg Oncol 2008; 98: 6-10.

5. Lee JH, Min YW, Lee JH, et al. Diagnostic group classifications of gastric neoplasms by endoscopic resection criteria before and after treatment: real-world experience. Surg Endosc 2015 Dec 22 [Epub]. http://dx.doi.org/10.1007/s00464-015-4710-z.

6. Lim H, Jung HY, Park YS, et al. Discrepancy between endoscopic forceps biopsy and endoscopic resection in gastric epithelial neoplasia. Surg Endosc 2014; 28: 1256-62.

7. Shin KY, Jeon SW, Cho KB, et al. Clinical outcomes of the endoscopic submucosal dissection of early gastric cancer are comparable between absolute and new expanded criteria. Gut Liver 2015; 9: 181-7.

8. Kim WH, Park CK, Kim YB, et al. A standardized pathology report for gastric cancer. Korean J Pathol 2005; 39: 106-13.

9. Kim L, Sakong J, Kim Y, et al. Developing the inpatient sample for the national health insurance claims data. Health Policy Manag 2013; 23: $152-61$. 
Appendix 1. Numbers of medical institutions according to administrative districts

\begin{tabular}{|c|c|c|c|}
\hline Administrative district & Tertiary hospital & General hospital & Other institutions (clinic) \\
\hline Seoul & 10 & 48 & $12,090(6,952)$ \\
\hline Busan & 3 & 24 & $3,567(2,048)$ \\
\hline Daegu & 1 & 11 & $2,542(1,538)$ \\
\hline Gwangju & 1 & 22 & $1,584(862)$ \\
\hline Daejeon & 1 & 8 & $1,599(981)$ \\
\hline Ulsan & 0 & 4 & $1,012(546)$ \\
\hline Gyeonggi-do & 1 & 55 & $10,424(5,883)$ \\
\hline Gangwon-do & 1 & 14 & $1,377(707)$ \\
\hline Chungcheongbuk-do & 1 & 10 & $1,488(791)$ \\
\hline Chungcheongnam-do & 0 & 12 & $2,019(1,013)$ \\
\hline Jeollabuk-do & 1 & 11 & $2,197(1,110)$ \\
\hline Jeollanam-do & 0 & 22 & 2,048 (913) \\
\hline Gyeongsangnam-do & 1 & 25 & $2,977(1,521)$ \\
\hline Jeju & 0 & 7 & $575(330)$ \\
\hline Sejong-si & 0 & 0 & $105(57)$ \\
\hline Total & 23 & 307 & $50,868(27,846)$ \\
\hline
\end{tabular}

